\title{
Role of Computed Tomography in Imaging and Classification of Acute Pancreatitis based on Revised Atlanta Classification: A 1-year Hospital-based Cross-sectional Study
}

\author{
Virupaxi. V. Hattiholi ${ }^{1}$, Sandhya A Yadav ${ }^{2}$ \\ ${ }^{1}$ Associate Professor, ${ }^{2}$ Senior Resident, Department of Radiodiagnosis, Jawaharlal Nehru Medical College, Nehru Nagar KLE \\ University, Belagavi 590010, Karnataka, India
}

Corresponding author: Dr. Virupaxi. V. Hattiholi, Department of Radiodiagnosis, Jawaharlal Nehru Medical College, Nehru Nagar KLE University, Belagavi 590010, Karnataka, India

DOI: 10.21276/ijcmsr.2018.3.2.34

How to cite this article: Virupaxi. V. Hattiholi, Sandhya A Yadav. Role of computed tomography in imaging and classification of acute pancreatitis based on revised atlanta classification: a 1-year hospital-based crosssectional study. International Journal of Contemporary Medicine Surgery and Radiology. 2018;3(2):B143-B146.

\section{A B S T R A C T}

Introduction: Several ideal multifactorial clinical and radiological scoring systems such as Ranson's criteria, the acute physiology and chronic health evaluation, and computed tomography (CT) severity index have developed to differentiate the patients with mild pancreatitis to those with severe cases. However, all these scoring systems has its own limitations. Hence, 1992 Atlanta Classification System, revised in 2012, is used to identify two phases of acute pancreatitis (AP) that is early and late. The current study aimed to classify AP based on revised Atlanta classification with the help of CT.

Material and Methods: A total of 60 patients with AP referred for CT scan were studied. Demographics, history and clinical investigations of the patients were recorded. A 64-section multiple detector CT scanner was used for the imaging. The images obtained were examined for the features of AP and classified based on revised Atlanta classification.

Results: Most $(29,48.33 \%)$ of the patients had interstitial edematous pancreatitis at initial stage. Most of the patients had irregular contour $(58,96.67 \%)$, diffuse size $(53,88.33 \%)$, homogenous density $(35,58.33 \%)$, necrosis $(36,60 \%)$, normal vascular structure $(49,81.67 \%)$, normal liver $(8,13.33 \%)$, sludge gallbladder $(1,1.67 \%)$, and no gall stones (50, $83.33 \%)$. Also, majority $(16,26.67 \%)$ of the patients had interstitial edematous pancreatitis with pseudocyst as late diagnosis.

Conclusion: The revised Atlanta Classification of AP has been able to provide complex patterns of the dynamics involved in the evolution of AP. It provides clear distinction between an early phase and a late phase.

Keywords: Acute Pancreatitis; Computed Tomography; Revised Atlanta Classification; Interstitial Edematous Pancreatitis; Pseudocyst

\section{INTRODUCTION}

One of the most common causes of acute abdomen is acute pancreatitis (AP). A clinically severe AP associated with local and systemic complications resulting in high morbidity and mortality develops in approximately 10-20\% of patients with AP. ${ }^{1,2}$ Patients can be treated effectively with early diagnosis of AP and early prediction of severe $\mathrm{AP}^{3}$ As there is a lack of a gold standard test, the diagnosis of AP remains problematic. According to the revised Atlanta classification ${ }^{4,5}$, three features are required for the diagnosis of AP: (1) Typical abdominal pain consistent with AP (acute onset of a severe, persistent, epigastric pain that may or may not come back), (2) Serum lipase or amylase activity $>3$ times the upper limit of normal, and (3) characteristic findings of AP on ultrasound, computed tomography (CT), magnetic resonance imaging, or. ${ }^{1}$ Although characteristic abdominal pain and increased laboratory values are readily used to make the diagnosis of AP, atypical presentations such as nonspecific upper abdominal pain or normal to increased amylase levels,
( $<3$ times normal) are often seen in the early stages (first $24 \mathrm{~h}$ of hospital admission). ${ }^{6}$ Early imaging is required to confirm or to exclude the diagnosis of AP as the serum amylase activity, which is used as a common biomarker, may be normal in 19$32 \%$ of cases during hospital admission and can be increased in case of other intra-abdominal inflammatory conditions. ${ }^{7}$ AP is classified into mild and severe pancreatitis. The interstitial pancreatitis, also called as mild pancreatitis, is linked with minimum organ failure and ordinary recovery. ${ }^{8}$ Severe pancreatitis, also called as necrotizing pancreatitis, is associated with local complications or organ failure, includes infection, necrosis or pseudocyst formation. ${ }^{9}$ Both the cases have different operating conditions in which the AP responds comparatively well to supportive therapy while severe pancreatitis often requires special monitoring and specific therapies with guarded prognosis. ${ }^{10}$

For the several manifestations of AP, a universally applicable classification system was introduced in 1992-Atlanta classification-for AP. ${ }^{9}$ The classification system was basically introduced to assess the treatment of the various collections 
of fluid, which were diagnosed during the course of AP. A significant advancement that was developed to differentiate amongst mild, moderate and severe forms of pancreatitis is the CT severity index. It determines the presence and the progress of pancreatic inflammation as well as necrosis. The Atlanta classification, useful to classify AP, was revised in 2008 and focused on morphologic criteria of defining the manifestations of AP, as outlined by means of CT. To update the terminology and to provide functional, clinical, and morphological classifications, the Atlanta classification was modified in 2012. ${ }^{11}$ This was to address the clinical course and the severity of the disease, differentiate early and late phase, stress on systemic inflammatory response syndrome, and multi organ failure. However, no such Indian studies on clinical and CT scan correlation of AP have been conducted. Considering these facts, the present study was undertaken to classify AP based on revised Atlanta classification with the aid of CT.

\section{MATERIAL AND METHODS}

This 1-year from (January 2015 - December 2015) hospitalbased cross-sectional study was conducted at the Department of Radio-diagnosis. Patients > 18 years and clinically suspected with AP with elevated levels of serum amylase and lipase levels were included in the study. Patients $<18$ years of age were exempted from the study. Patients fulfilling the selection criteria were referred for the $\mathrm{CT}$ scan. The calculated sample size was based on $80 \%$ average number of cases admitted with AP in the previous 3 years. All 60 patients who fulfilled the selection criteria were finally enrolled in the study. The ethical clearance certificate was attained from the Institutional Ethics Committee prior to initiation of the study. All the selected patients were explained about the study and a written informed consent was obtained.

\section{Data collection}

A descriptive data of the patients such as age, sex, and detailed history were obtained along with their clinical history followed by the recording of the finding of clinical examination in study participants.

\section{Study procedure}

A 64-section multiple detector computer tomography (MDCT) scanner manufactured by Siemens was used for the imaging. A power injector (dual syringe injector) was used to inject $80-100 \mathrm{~mL}$ of nonionic iodinated contrast (iodine concentration, $400 \mathrm{mg}$ ) at $2.5-3 \mathrm{~mL} / \mathrm{s}$, followed by acquiring the images after 13-20 s for arterial phase and after 40-60 s for portal venous phase. A $900-1000 \mathrm{~mL}$ of water was diluted with $3 \%$ diatrizoate meglumine, and diatrizoate sodium was administered orally to the patients almost 30-45 min prior to scan the outline of gastrointestinal tract. AP was graded by the modified CT severity index, depending upon the contrast-enhanced computed tomography (CECT). Patients were followed up till they were discharged based on their clinical outcome. The images obtained were examined for the features of AP and classified based on revised Atlanta classification.

\section{Statistical analysis}

The data pooled were coded and entered Microsoft Excel
Worksheet. The categorical data was expressed as rates, ratios and proportions. The continuous data was expressed as mean \pm standard deviation (SD).

\section{RESULTS}

In this study, of 60 patients, 51 (85\%) were men and only 9 were women and most of the study population $(31,51.67 \%)$

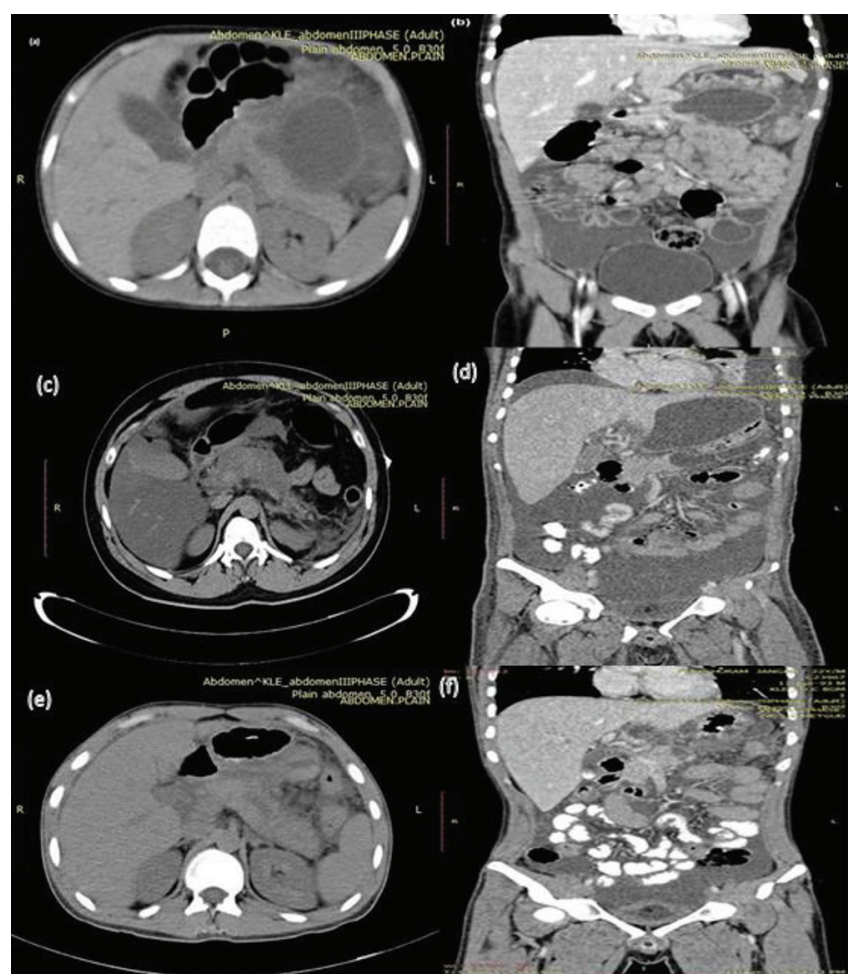

Figure-1: Computed tomography images of patients with acute pancreatitis. (a) and (b): Interstitial edematous pancreatitis with pseudocyst; (c) and (d): Acute necrotizing pancreatitis with walled off necrosis; (e) and (f): Acute necrotizing pancreatitis with acute necrotic fluid collection

\begin{tabular}{|l|c|}
\hline Variable & $\mathbf{n},(\%)$ \\
\hline Sex & $51(85)$ \\
\hline Male & $9(15)$ \\
Female & $31(51.67)$ \\
\hline Age & $26(43.33)$ \\
\hline $18-30$ & $3(5.00)$ \\
$31-40$ & $38(63.33)$ \\
$41-50$ & $22(36.67)$ \\
\hline History of alcohol consumption \\
\hline Yes \\
No & $43(71.67)$ \\
\hline Clinical presentation & $41(68.33)$ \\
\hline Severe epigastric pain & $33(55.00)$ \\
Nausea & $22(36.67)$ \\
Vomiting & $16(26.67)$ \\
Fever & $15(25)$ \\
Breathlessness & \\
Chills & $17(28.33)$ \\
\hline Chest radiography & $43(71.67)$ \\
\hline Normal \\
Pleural effusion
\end{tabular}




\begin{tabular}{|c|c|c|}
\hline Parameters & $\begin{array}{l}\text { First, } \\
\mathrm{n}(\%)\end{array}$ & $\begin{array}{l}\text { Follow-up, } \\
n(\%)\end{array}$ \\
\hline \multicolumn{3}{|l|}{ Contour } \\
\hline Irregular & $58(96.67)$ & $58(96.67)$ \\
\hline Regular & $2(3.33)$ & $2(3.33)$ \\
\hline \multicolumn{3}{|l|}{ Size } \\
\hline Diffuse & $53(88.33)$ & $53(88.33)$ \\
\hline Focal & $7(11.67)$ & 7 (11.67) \\
\hline \multicolumn{3}{|l|}{ Density } \\
\hline Heterogeneous & $24(40)$ & $25(41.67)$ \\
\hline Homogenous & $36(60)$ & $35(58.33)$ \\
\hline \multicolumn{3}{|l|}{ Necrosis } \\
\hline Yes & $35(58.33)$ & $24(40)$ \\
\hline No & $25(41.67)$ & $36(60)$ \\
\hline \multicolumn{3}{|l|}{ Gall bladder } \\
\hline Sludge & $1(1.67)$ & $1(1.67)$ \\
\hline Normal & $55(91.67)$ & $9(15)$ \\
\hline No & $4(6.67)$ & $50(83.33)$ \\
\hline \multicolumn{3}{|l|}{ Gall stones } \\
\hline No stones & $50(83.33)$ & $50(83.33)$ \\
\hline Sludge & $1(1.67)$ & $1(1.67)$ \\
\hline Stone & $9(15)$ & $9(15)$ \\
\hline \multicolumn{3}{|l|}{ Vascular structure } \\
\hline Normal & $51(85)$ & $49(81.67)$ \\
\hline Portal and splenic vein thrombosis & $4(6.67)$ & $4(6.67)$ \\
\hline Portal vein thrombosis & $4(6.67)$ & $2(3.33)$ \\
\hline Splenic vein thrombosis & $1(1.67)$ & $5(8.33)$ \\
\hline \multicolumn{3}{|l|}{ Liver } \\
\hline Fatty liver & $5(8.33)$ & $5(8.33)$ \\
\hline Hepatomegaly & $13(21.67)$ & $11(21.67)$ \\
\hline Hepatomegaly with fatty infiltra- & $8(13.33)$ & $1(1.67)$ \\
\hline $\begin{array}{l}\text { tion } \\
\text { Normal }\end{array}$ & $34(56.67)$ & $34(56.67)$ \\
\hline \multicolumn{3}{|l|}{ Others } \\
\hline Pancreatic fat standing & 59(98.33) & $58(96.67)$ \\
\hline Peripancreatic fluid collection & $46(76.67)$ & $44(73.33)$ \\
\hline Pseudoscyst & $1(1.67)$ & $14(23.33)$ \\
\hline Wall of necrosis & $9(15)$ & $13(21.67)$ \\
\hline Ascites & $44(73.33)$ & $22(36.67)$ \\
\hline Parenchymal involvement & $44(73.33)$ & $17(28.33)$ \\
\hline
\end{tabular}

belonged to the age-group of 18-30 years. History of alcohol consumption was noted in $63.33 \%$ of the patients. A total of $43(71.67 \%)$ patients presented with severe epigastric pain and, 35 (58.33\%) patients reported with increased total count. Increased renal function and liver function was reported in $19(31.67 \%)$ and $5(8.33 \%)$ patients, respectively. Baseline characteristics of the patients are shown in Table 1. Majority of the patients presented with increased serum amylase (49, 81.67\%) and increased serum lipase (59, 83.33\%). Chest radiography revealed pleural effusion in $43(71.67 \%)$ patients. CT scan findings during first and follow-up is shown in Table 2. Among 60 patients, 58 (96.67\%) had irregular contour, 53 $(88.33 \%)$ had diffuse size. Homogenous density, and necrosis was observed in $36(60 \%)$ and in $35(58.333 \%)$ patients, respectively. However, $34(56.67 \%)$ patients had normal liver, $1(1.67 \%)$ had sludge gallbladder, and no gall stones was observed in in $50(83.33 \%)$ patients. The follow-up CT scan

\begin{tabular}{|l|c|c|}
\hline Diagnosis & $\begin{array}{c}\text { First, } \mathbf{n}, \\
\text { (\%) }\end{array}$ & Follow-up \\
\hline Interstitial edematous pancreatitis & $29(48.33)$ & $8(13.33)$ \\
\hline Acute necrotizing pancreatitis & $10(16.67)$ & 0 \\
\hline $\begin{array}{l}\text { Acute necrotizing pancreatitis with } \\
\text { acute necrotic fluid collection }\end{array}$ & $10(16.67)$ & $7(11.67)$ \\
\hline $\begin{array}{l}\text { Interstitial edematous pancreatitis } \\
\text { with acute peripancreatic fluid } \\
\text { collection }\end{array}$ & $5(8.33)$ & $8(13.33)$ \\
\hline $\begin{array}{l}\text { Acute necrotizing pancreatitis with } \\
\text { walled off necrosis }\end{array}$ & $5(6.67)$ & $15(25)$ \\
\hline $\begin{array}{l}\text { Interstitial edematous pancreatitis } \\
\text { with pseudocyst }\end{array}$ & $1(1.67)$ & $16(26.67)$ \\
\hline $\begin{array}{l}\text { Acute necrotizing pancreatitis with } \\
\text { walled off necrosis and Infection }\end{array}$ & 0 & $2(3.33)$ \\
\hline \multicolumn{2}{|c|}{ Spontaneous reduction } & - (6.67) \\
\hline $\begin{array}{l}\text { Table-3: Distribution of study population according to the final } \\
\text { diagnosis based on first and follow-up CT scan findings }\end{array}$ \\
\hline \multicolumn{2}{|l|}{} \\
\hline
\end{tabular}

of the study population revealed almost similar findings as that of the first CT scan findings. It was observed that most $(58,96.67 \%)$ of the patients had irregular contour, followed by diffuse size in 53 (88.33\%), homogenous density in 35 (58.33\%), necrosis in $24(40 \%)$, normal vascular structure in $49(81.67 \%)$, normal liver in $8(13.33 \%)$, sludge gallbladder in $1(1.67 \%)$, and no gall stones in $50(83.33 \%)$ patients.

CT scan findings during first and follow-up is shown in Table 3. Out of 60 patients, 29 (48.33\%) patients were diagnosed of intestinal edematous pancreatitis. Other diagnosis is shown in Table 3. Final diagnosis revealed that most of the patients had intestinal edematous pancreatitis with pseudocyst in 16 (26.67\%) patients, followed by acute necrotizing pancreatitis with walled off necrosis in 15 (25\%) patients. (Fig. 1)

\section{DISCUSSION}

The present study reported male preponderance among the study population. This suggested the prevalence of AP among men. A study by $\mathrm{Yu}$ Shi et al. ${ }^{1}$ reported 42 (55\%) men patients. In this study, the age of the study population ranged from 18 to 48 years and more than half of the population $(31,51.67 \%)$ presented with the age between 18 and 30 years. This showed the prevalence of AP in young population. In contrast, a study by Bollen et al. ${ }^{12}$ reported mean age of 53 years. The present study reported history of alcohol consumption in two-third of the study population $(38,68.33 \%)$. However, the study conducted by Bollen et al. ${ }^{12}$ reported alcohol abuse in $22 \%$ of the cases. The higher rate of alcohol consumption observed in the present case was due to the male preponderance and younger age of the study population. ${ }^{12} \mathrm{~A}$ higher rate of severe epigastric pain was reported as the commonest clinical presentation in 43 (71.67\%) patients.

Based on the computed tomographic features identified in patients, most of the patients had interstitial edematous pancreatitis. This was similar to the study by Surekha et al. ${ }^{13}$, who reported 80 to $90 \%$ of patients with interstitial edematous pancreatitis, which is a milder variant and characterized by the absence of pancreatic or peripancreatic necrosis on imaging. An amount of inflammatory haziness 
or mild stranding is present in peripancreatic fat and usually resolves itself within a week. ${ }^{14}$ The revised Atlanta classification defines four types of collections based on the type of AP. Two types of collections are associated with acute interstitial edematous pancreatitis: Acute peripancreatic fluid collections (APFC) and pseudocyst. The other two collections are associated with necrotizing pancreatitis-acute necrotic collection (ANC) and walled-off-necrosis (WON). Most of the collection of acute fluid are generally uninfected; however, if persists for a long time, it is likely to develop into pancreatic pseudocyst. ${ }^{13}$ The revised classification helps to describe patients with AP precisely, standardize terminology and aid in treatment planning. It also aids in describing AP as necrotizing pancreatitis and differentiates between an early phase ( $1^{\text {st }}$ week) and a late phase (after the $1^{\text {st }}$ week). The clinical parameters are used to define the first phase while CECT finding along with clinical staging is used to define the second phase morphologically. ${ }^{13}$

Differentiation of APFC and acute necrotizing collection (ANC) generally becomes difficult during the initial 1-2 weeks on imaging, as collections may be shown with fluid density without any solid component. However, the collection might get evident after first week. Walled-off pancreatitis necrosis (WON) generally had a well-defined and reactive wall. ${ }^{13}$ The collections might be sterile or infected based on the suspected existence of gas within the collection. Infection in all the four types of collections can be detected by a non-invasive diffusion-weighted (DW) MRI, in which the infected collection shows peripheral bright signals on DW-MRI images and diffuse restriction is shown in sterile collections. Infections are most often seen with ANC and WON. Thus, collections that are associated with necrotizing pancreatitis mostly require drainage and surgery. ${ }^{11,15,16}$

Overall, the revised Atlanta Classification of AP provides complex patterns of the dynamics involved in the evolution of AP.

\section{CONCLUSION}

Based on the findings of this study, it may be concluded that most of the patients had interstitial edematous pancreatitis at initial stage. Based on the follow-up CT scan findings, most of the patients had interstitial edematous pancreatitis with pseudocyst as late diagnosis. The revised Atlanta Classification of AP provides complex patterns of the dynamics involved in the evolution of AP. It precisely describes patients with AP, which helps in the treatment planning. Furthermore, it provides clear distinction between an early phase (1st week) and a late phase (after the 1st week). The clinical parameters are defined under first phase, while morphologically based on CECT findings combined with clinical staging under second phase. The most vital change is the precise recognition of various pancreatic collections. Overall the revised Atlanta classification with CT not only helps to guide the management but also to monitor the success of treatment.

\section{REFERENCES}

1. Shi Y, Liu Y, Liu Yq, Gao F, Li Jh, Li Qj, et al. Early diagnosis and severity assessment of acute pancreatitis
(AP) using MR elastography (MRE) with spin-echo echo-planar imaging. J Magn Reson Imaging 2017.

2. Forsmark CE, Baillie J. AGA Institute technical review on acute pancreatitis. Gastroenterol 2007;132(1): 20222044.

3. Peery AF, Dellon ES, Lund J, Crockett SD, McGowan $\mathrm{CE}$, Bulsiewicz WJ, et al. Burden of gastrointestinal disease in the United States: 2012 update. Gastroenterol 2012;143 (3): 1179-1187. e3.

4. Banks P, Bollen T, Dervenis C, Gooszen H, Johnson C, Sarr M, et al. Acute Pancreatitis Classification Working Group: Classification of acute pancreatitis-2012: revision of the Atlanta classification and definitions by international consensus. Gut 2013;62 (5): 102-111.

5. Lerch MM. Classifying an unpredictable disease: the revised Atlanta classification of acute pancreatitis. Gut 2013;62 (2): 2-3.

6. Casas JD, Díaz R, Valderas G, Mariscal A, Cuadras P. Prognostic value of CT in the early assessment of patients with acute pancreatitis. Am J Roentgenol 2004;182 (4): 569-574.

7. Matull W, Pereira S, O'donohue J. Biochemical markers of acute pancreatitis. J Clin Pathol 2006;59 (6): 340344.

8. van Grinsven J, van Vugt JL, Gharbharan A, Bollen TL, Besselink MG, van Santvoort HC, et al. The Association of Computed Tomography-Assessed Body Composition with Mortality in Patients with Necrotizing Pancreatitis. J Gastrointest Surg 2017: 1-9.

9. Bradley EL. A clinically based classification system for acute pancreatitis: summary of the International Symposium on Acute Pancreatitis, Atlanta, Ga, September 11 through 13, 1992. Arch Surg 1993;128 (7): 586-590.

10. De Beaux A, Palmer K, Carter D. Factors influencing morbidity and mortality in acute pancreatitis; an analysis of 279 cases. Gut 1995;37 (5): 121-126.

11. Thoeni RF. The revised Atlanta classification of acute pancreatitis: its importance for the radiologist and its effect on treatment. Radiol 2012;262 (1): 751-764.

12. Bollen TL, Singh VK, Maurer R, Repas K, Van Es HW, Banks PA, et al. Comparative evaluation of the modified CT severity index and CT severity index in assessing severity of acute pancreatitis. Am J Roentgenol 2011;197 (4): 386-392.

13. Sureka B, Bansal K, Patidar Y, Arora A. Imaging lexicon for acute pancreatitis: 2012 Atlanta Classification revisited. Gastroenterol Rep 2016;4 (3): 16-23.

14. Singh VK, Bollen TL, Wu BU, Repas K, Maurer R, $\mathrm{Yu}$ S, et al. An assessment of the severity of interstitial pancreatitis. Clin Gastroenterol Hepatol 2011;9 (1): 1098-1103.

15. Murphy KP, O'Connor OJ, Maher MM. Updated imaging nomenclature for acute pancreatitis. Am J Roentgenol 2014;203 (5): W464-W469.

16. Islim F, Salik AE, Bayramoglu S, Guven K, Alis H, Turhan AN. Non-invasive detection of infection in acute pancreatic and acute necrotic collections with diffusionweighted magnetic resonance imaging: preliminary findings. Abdom Imaging 2014;39 (5): 472-481.

Source of Support: Nil; Conflict of Interest: None

Submitted: 06-05-2018; Accepted: 08-06-2018; Published online: 19-06-2018 